\title{
Uso da técnica TightRope modificada em cães com ruptura do ligamento cruzado cranial $^{*}$
}

\section{Use of modification TightRope technique in dogs with rupture of cranial crucial ligament}

\author{
Thales Bregadioli, ${ }^{* \star}$ Francisco Cláudio Dantas Mota, ${ }^{* \star *}$ Duvaldo Eurides, ${ }^{* *}$ Laura Melgaço Faria, ${ }^{* *}$ Raíra Costa Dias, ${ }^{* *}$ \\ Luiz Augusto de Souza****
}

\begin{abstract}
Resumo
A ruptura do ligamento cruzado cranial (RLCCr) figura entre as principais enfermidades ortopédicas na espécie canina, sendo responsável por instabilidade da articulação do joelho, o que resulta em claudicação e desenvolvimento de doença articular degenerativa (DAD). O objetivo deste trabalho foi avaliar os resultados da técnica de "TightRope" modificada (TRM) na estabilização do joelho de cães com ruptura do ligamento cruzado cranial (RLCCr). O procedimento cirúrgico foi realizado em seis animais, os quais foram avaliados por 120 dias de pós-operatório. A modificação da técnica para estabilização extracapsular da RLCCr, consiste na utilização de fio de fluorcarbono, e fio de aço inoxidável de $1 \mathrm{~mm}$ como forma de fixação óssea. A "TRM" promoveu a estabilidade do joelho dos pacientes com ruptura do ligamento cruzado cranial no pós-operatório imediato e durante o acompanhamento clínico pós-cirúrgico demonstrou, aos 30 dias, progressiva melhora da marcha e suporte de peso à deambulação, estando todos os animais recuperados clinicamente aos 120 dias de pós-operatório.
\end{abstract}

Palavras-chave: joelho, cão, instabilidade, fluorcarbono.

\begin{abstract}
Rupture of the cranial cruciate ligament is one of the leading orthopedic diseases in dogs. Thus aimed to evaluate the technique of "Tightrope" modified (TRM) in the knees of dogs with the purpose of providing less invasive and cost-effective surgery. The surgical procedure was performed in six animals, and these were evaluated up to 120 days postoperatively. The modification of the technique for stabilization of extra capsular CCL consists in using fluorocarbon and the stainless steel wire of $1 \mathrm{~mm}$ in order bone fixation. The "TRM" promoted the stability of the knee of patients diagnosed with cranial cruciate ligament rupture in the immediate postoperative period. Clinical follow-up after surgery showed after 30 days postoperatively, improved gait and progressive weight support for ambulation, being all animals recovered clinically at 120 days postoperatively.
\end{abstract}

Keywords: knee, dog, instability; fluorocarbon.

\section{Introdução}

A ruptura do LCCr é a enfermidade ortopédica do joelho mais importante da espécie canina, e a mais encontrada (Hayashi et al., 2003), sendo considerada a terceira maior causa de problemas ortopédicos desta espécie (Souza et al., 2011).

Os ligamentos do joelho são responsáveis pela sustentação da articulação e especificamente, os ligamentos cruzados cranial e caudal possuem importância na estabilização do movimento craniocaudal da articulação, além da limitação da rotação interna da tíbia em relação ao fêmur por parte do LCCr (Durana, 2009).

(Hulse (1995) e Canapp (2007), descrevem que o ligamento cruzado cranial é composto de duas porções: as faixas craniomedial e craniolateral. A primeira apresenta-se tensa tanto a flexão quanto na extensão, e a segunda unicamente na extensão, tornando-se relaxada durante a flexão. Este fato faz com que a faixa cranio- medial seja responsável pelo controle primário contra o movimento de gaveta cranial, impedindo a hiperextensão do joelho.

A RLCCr em cães é uma doença multifatorial envolvendo fatores genéticos, conformacionais e inflamatórios que, em conjunto, criam um desequilíbrio entre a força biomecânica aplicada no ligamento e sua habilidade em sustentar essa carga, levando, eventualmente, à ruptura e à instabilidade articular (Griffon, 2010; Comerford et al., 2011). Sendo assim, sua origem pode ser por determinação traumática e/ou degenerativa, podendo o animal apresentar claudicação e dor de intensidade variável à manipulação articular (Durana, 2009).

Esta enfermidade pode afetar animais de qualquer idade, sexo e/ou raça, porém é mais comum em raças de grande porte, como rottweiler, bullmastiff, chow-chow (Whitehair e Vasseur, 1993).

O teste "movimento de gaveta", um sinal patognomônico da lesão, caracteriza-se pela translação cranial da tíbia em relação

\footnotetext{
${ }^{*}$ Recebido em 20 de janeiro de 2014 e aceito em 16 de maio de 2014.

${ }^{* *}$ Médico veterinário. Residente. Hospital veterinário - UFU.

${ }^{* * *}$ Médico veterinário. Professor Doutor. Faculdade de Medicina Veterinária/UFU.

${ }^{* * * \star M e ́ d i c o ~ v e t e r i n a ́ r i o . ~ P r o f e s s o r ~ D o u t o r . ~ E s c o l a ~ d e ~ V e t e r i n a ́ r i a / U F G ~}$

Autor para contato: thalesbregadioli@hotmail.com.
} 
ao fêmur, possibilitando o diagnóstico clínico para RLCCr (Palmer, 2005). O movimento de gaveta deve ser realizado com o membro pélvico flexionado e também em extensão (Vasseur, 2003). A sensibilidade do teste aumenta consideravelmente quando realizado sob anestesia, reduzindo o risco de resultados falso-negativos (Carobbi e Ness, 2009). De acordo com Moore e Read (1996), para que o teste seja considerado positivo, o deslocamento cranial da tíbia em relação ao fêmur deve ser superior a $2 \mathrm{~mm}$.

Por meio da manobra de compressão da tíbia, é também possível detectar o movimento cranial da tíbia, associado à ruptura total do LCCr (Fossum, 2002). A demonstração do deslocamento cranial da tíbia em relação ao fêmur por meio da realização do exame radiográfico, concomitante ao teste de compressão tibial, é uma opção para a confirmação da RLCCr (Palmer, 2005).

Existem várias formas de tratar a RLCCr, entretanto, a escolha terapêutica é influenciada pela idade, tamanho corporal e função do animal, além das considerações econômicas do proprietário (Vasseur, 2003).

O tratamento clínico conservador consiste basicamente em restrição da atividade física, controle alimentar para redução de peso corporal, analgesia, fisioterapia e, eventualmente, aplicação de bandagem em cães até $10 \mathrm{~kg}$ de peso corporal (Piermattei, et al., 2006; Comerford, 2007). Observa-se, no entanto, que as lesões tardias de meniscos têm ocorrido, sobretudo, em cães pequenos (Durana, 2009). Evolução dos sinais clínicos e osteoartrose gradativa são observados sobretudo em cães entre 15 - 20kg, submetidos ao tratamento conservador (Schultz, 2007).

De acordo com Kim et al., (2008), a estabilização cirúrgica deve ser recomendada a todos os pacientes, principalmente aos cães com peso corporal superior a 10 e $15 \mathrm{~kg}$.

As técnicas cirúrgicas que estabilizam a articulação do joelho são divididas em três grupos principais: método extracapsular, método intracapsular e as osteotomias proximais à tíbia (Piermattei, et al., 2006; Kim et al., 2008). Entretanto, não há uma técnica padrão, sendo de responsabilidade do cirurgião a escolha do procedimento favorável a cada situação (Conzemius et al., 2005).

Outra opção cirúrgica são as próteses para substituição total do joelho de cães com doença articular degenerativa (DAD), cujos resultados de aplicação têm demonstrado viabilidade para cães com peso corporal entre 12 e 60kg (Liska e Doyle, 2009).

As técnicas extracapsulares requerem menos instrumentos especializados, são geralmente mais simples e rápidas, apresentam melhor custo-benefício e são mais acessíveis (Jerram e Walker, 2003). Um dos métodos mais utilizados é a sutura fabelo-tibial lateral, independentemente do tamanho do animal; o fio, geralmente, é passado com agulha curva ao redor do ligamento fabelofemoral e por um orifício criado na crista tibial. A técnica de sutura fabelotibial lateral é tecnicamente menos complexa do que métodos intracapsulares ou osteotomias e, por isso, continuam sendo praticadas por muitos cirurgiões. A melhor sutura lateral é aquela que elimina o deslocamento crânio da tíbia em relação ao fêmur, cuja colocação não interfira na distância entre os pontos de ancoragem durante os movimentos de flexão e extensão (Hulse et al., 2010). Porém, independentemente do ponto exato de fixação da sutura lateral, mudanças consideráveis na tensão desta podem ocorrer durante o movimento da articulação. Essa mudança na tensão aumenta significativamente com a flexão da articulação, podendo neste momento exercer excessiva pressão sobre o compartimento lateral do joelho, causando possível desequilíbrio na distribuição de carga das superfícies articulares e dos meniscos (Fischer, et al., 2010; Tonks et al., 2010).

Recentemente desenvolveu-se na Medicina Veterinária a técnica "TightRope", para estabilização da articulação do joelho de cães com RLCCr. Esta técnica foi baseada na estabilização da articulação tibiotársica aplicada em humanos. A "TightRope" mostrou-se uma técnica minimamente invasiva para estabilização de joelhos, na qual por meio da confecção de túneis ósseos, permite-se a inserção de um mecanismo extracapsular para estabilização do LCCr. Com isso, anula-se o movimento de gaveta com menor risco de ocorrência de complicações graves (Cook et al., 2010).

A técnica de "TightRope" buscou, com sucesso, aperfeiçoar a estabilização extracapsular do joelho acometido pela ruptura do ligamento cruzado cranial, colocando em pontos isométricos um implante de maior resistência e rigidez com fixação óssea entre a tíbia e o fêmur, especificamente para reparação da RLCCr (Tight Rope CCI ${ }^{\circledR}$, Arthrex Vet Systems, Naples, FL, USA., 2008) (Figura 1).

Figura 1: Esquema da técnica "TightRope" original mostrando os túneis ósseos em pontos isométricos. A, vista cranial. B, vista lateral. C, vista medial. Fonte: adaptado de ARTHREX VET SYSTEMS 2008

A fita de TightRope, produzida pela Arthrex Vet Systems ${ }^{\circledR}$, é altamente resistente, composta por uma cadeia longa de ultrapolietileno com alto peso molecular, o que lhe dá uma força insuperável, praticamente eliminando a possibilidade de sua ruptura (Richard et al., 2008).

Devido à necessidade da criação de túneis ósseos largos, a técnica é indicada para animais com peso corpóreo superior ou igual a $15 \mathrm{~kg}$. A aplicação dessa técnica em cães com ruptura do LCCr tem proporcionado bons resultados e, quando comparada com a "Osteotomia niveladora do platô tibial" (TPLO), resulta menor tempo de anestesia e cirurgia, apresentando menor taxa de complicações pós-operatórias (Cook et al., 2010).

O objetivo deste trabalho foi avaliar a efetividade, bem como a resposta clínica, da estabilização de joelhos de seis cães com ruptura do LCCr, atendidos no Hospital Veterinário da 
Universidade Federal de Uberlândia, com peso variando de 5 a $32 \mathrm{~kg}$ e idade de três a sete anos, por meio da técnica "TightRope" que foi modificada utilizando-se fio de fluorcarbono, e fio de aço inoxidável.

\section{Relato dos casos}

Foram utilizados seis cães adultos da rotina clínica do Hospital Veterinário da Universidade Federal de Uberlândia, sendo dois machos e quatro fêmeas, com peso variando entre 5 e $32 \mathrm{~kg}$. Todos os cães apresentaram histórico de claudicação aguda no membro pélvico após exercício extenuante, com histórico de impotência funcional de início súbito.

O diagnóstico clínico de ruptura de ligamento cruzado cranial se deu através do teste de gaveta cranial que se mostrou positivo, além de confirmação radiológica na posição mediolateral, onde no momento da exposição ao raio-x promovia-se o teste de compressão tibial para demonstrar o deslocamento cranial do platô tibial em relação ao fêmur, sendo observado, em alguns casos, achados como interrupção da imagem do coxim adiposo, distensão da capsula articular, osteófitos periarticulares e esclerose do osso subcondral, que são alterações que podem ser visualizadas no exame radiográfico em animais com $\mathrm{RLCCr}$ (Oliveira, et al., 2009; Kealy, et al., 2011).

Após exames laboratoriais pré-cirúrgicos, como hemograma, ALT e creatinina com resultados favoráveis, os animais foram encaminhados ao setor de clinica cirúrgica para correção da ruptura do ligamento cruzado cranial utilizando a técnica de "TigthRope" modificada (TRM), com fio de fluorcarbono 0,6 mm e fio de aço inoxidável de 1,0 ou $1,5 \mathrm{~mm}$, de acordo com o peso do animal (Figura 2).

A utilização do fio de fluorcarbono e do fio de aço seguiu o seguinte critério: cães com peso inferior há $10 \mathrm{~kg}$ foi utilizado um fio fluorcarbono de $0,6 \mathrm{~mm}$ e fio de aço $1,0 \mathrm{~mm}$, animais com peso entre 10 e 25 quilos, dois fios fluorcarbono de $0,6 \mathrm{~mm}$ trançados e fio de aço $1,5 \mathrm{~mm}$ e em cães com peso superior a $25 \mathrm{~kg}$, três fios fluorcarbono de $0,6 \mathrm{~mm}$ trançados, também com a cavilha feita de fio de aço $1,5 \mathrm{~mm}$.

O fio de aço de 1,0 ou $1,5 \mathrm{~mm}$ era torcido em $360^{\circ}$ até atingir um formado semelhante a uma cavilha, suas dimensões variavam de 1 a $0,5 \mathrm{~cm}$ conforme o peso do animal, sendo utilizadas duas cavilhas para cada procedimento.

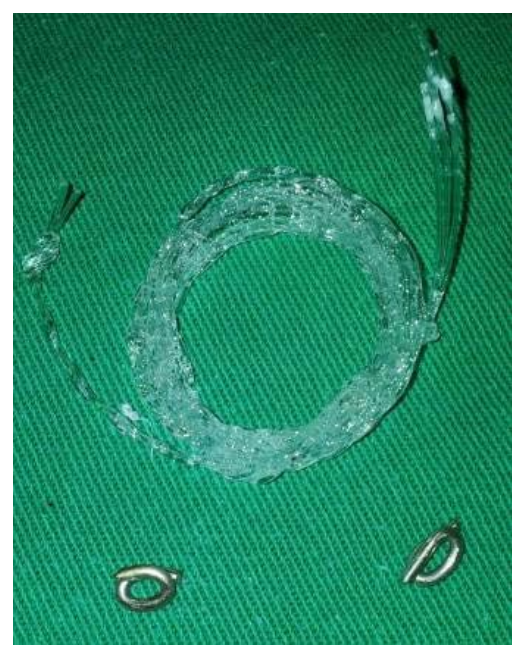

Figura 2: Imagem fotográfica mostrando dois fios de fluorcarbono 0,6 trançados e fios de aço inoxidavel de $1,5 \mathrm{~mm}$ retorcidos em $360^{\circ}$, utilizados na modificação da técnica de "TightRope".
Inicialmente à realização do procedimento, fazia-se uma artrotomia através de incisão parapatelar lateral de tamanho mínimo o suficiente para inspeção e se necessária, remoção de fragmentos dos meniscos lesionados e eventuais restos ligamentares rompidos.

Para a execução da técnica, foram realizados dois túneis ósseos com uma broca ortopédica cujo diâmetro variou de acordo com o tamanho do animal. O primeiro túnel foi feito na região cranioproximal ao sulco do tendão extensor digital longo progredindo através da tíbia, num ângulo ligeiramente craniodistal (Figura 3A)

O fio de fluorcarbono era passado pelo orifício da cavilha, depois as duas extremidades do fio eram transpassadas através do orifício feito na tíbia no sentido medial para lateral, fixando a cavilha e o fio na face medial da tíbia (Figura 3B). Em seguida um segundo túnel era criado cranial e distalmente à fabela lateral, na porção caudal do côndilo femoral, atravessando o fêmur distal e emergindo no lado medial do côndilo femoral, caudalmente ao músculo vastomedial (Figura 3C). Em seguida, as duas extremidades do fio eram transpassadas através da nova perfuração, do côndilo lateral para face medial (Figura 3D). Por fim, uma das extremidades do fio de fluorcarbono era passada no orifício da segunda cavilha, sendo fixada com um nó de cirurgião duplo, seguido de três nós simples, com o membro em posição ortostática (aproximadamente $135^{\circ}$ ) (Figura $3 E)$. Para facilitar a passagem dos fios de fluorcarbono pelos túneis ósseos, utilizou-se como guia, um fio de Kirchner $0,5 \mathrm{~mm}$ dobrado ao meio em 180 graus.

Imediatamente após a cirurgia, os membros operados eram avaliados clinicamente quanto ao movimento de gaveta cranial, sendo classificado em positivo ou negativo e, encaminhados para o exame radiográfico na posição mediolateral, com compressão tibial para verificar a posição do platô tibial em relação ao fêmur (Figura 4).

No acompanhamento clínico ao longo de 120 dias pósoperatórios, avaliou-se o grau de apoio do membro operado, atribuindo-se quatro categorias de acordo com Dias et al. (2009): excelente (4), nos casos em que havia total suporte do peso pelo animal e sem claudicação; bom (3), quando apresentava apenas claudicação discreta; satisfatório (2), na presença de claudicação moderada, mas com suporte de peso e; insatisfatório (1) quando podia ser notada claudicação permanente, sem suporte de peso.

O primeiro retorno dos animais ocorreu aos sete dias de pósoperatório.

Neste momento, foi observado que todos os pacientes mostravam-se tolerantes à manipulação cuidadosa do joelho, permitindo extensão e flexão, além de pouco ou nenhuma presença de edema articular. Três animais apresentaram-se com nota (2), os outros três, todos eles com menos de $15 \mathrm{~kg}$, apresentaram-se com nota (1).

No acompanhamento clínico, quatro animais, já aos 15 dias de pós-cirúrgico, apresentaram apoio do membro operado sendo classificados com nota (2), evoluindo para nota (3) aos 30 dias e nota (4) já aos 60 dias de pós-operatório. Entretanto, dois animais, com menos de 10 quilos, avaliados aos 15 dias, não apoiavam o membro operado, recebendo nota (1), somente aos 30 dias de pós-operatório demonstraram melhora clínica com nota (2), após 120 dias apresentaram-se com apoio e deambulação excelente, recebendo nota (4) (Tabela 1). 


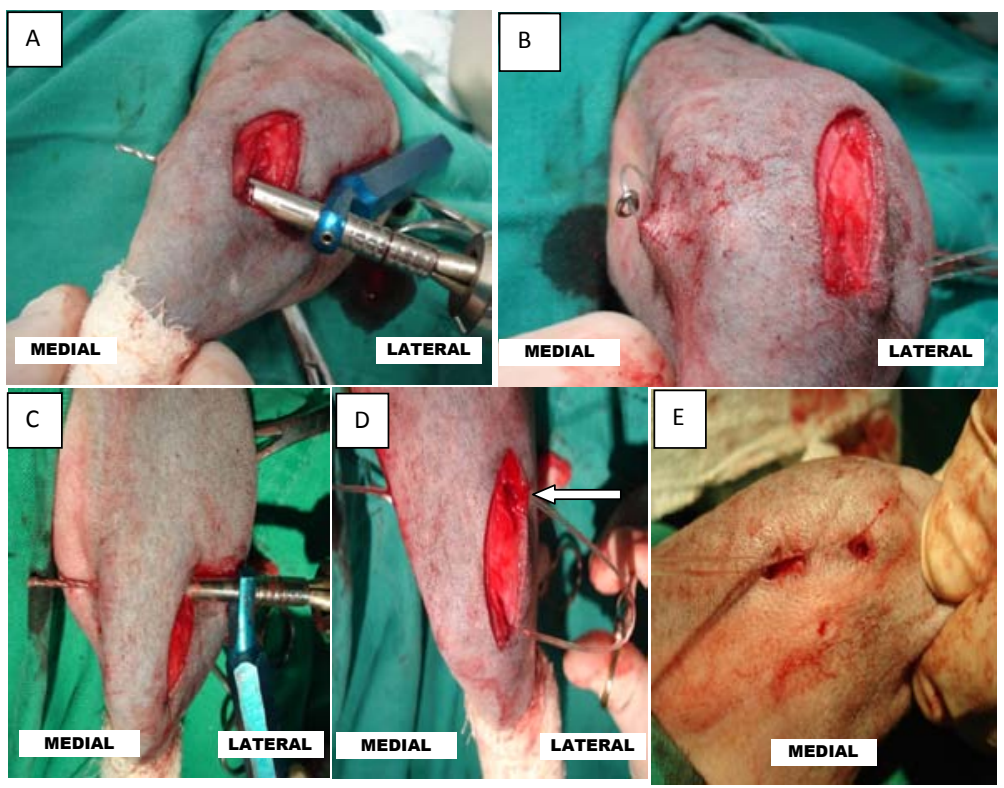

Figura 3: Imagens fotográficas trans-operatórias da estabilização da articulação do joelho com a técnica de "TightRope" modificada. A- Túnel realizado na crista da tíbia, na região crânio-proximal ao sulco do tendão extensor digital longo progredindo através da tíbia, sentido lateromedial. B- Fio de fluorcarbono fixado a cavilha, e transpassado através do orifício feito na tíbia no sentido medial para lateral. C- Túnel criado cranial e distalmente à fabela lateral, na porção caudal do côndilo femoral, atravessando o fêmur distal e emergindo no lado medial do côndilo femoral. D- Fio de fluorcarbono transpassado através da nova perfuração (seta), do côndilo lateral para face medial. E - Aspecto da face medial do membro após a fixação da segunda cavilha
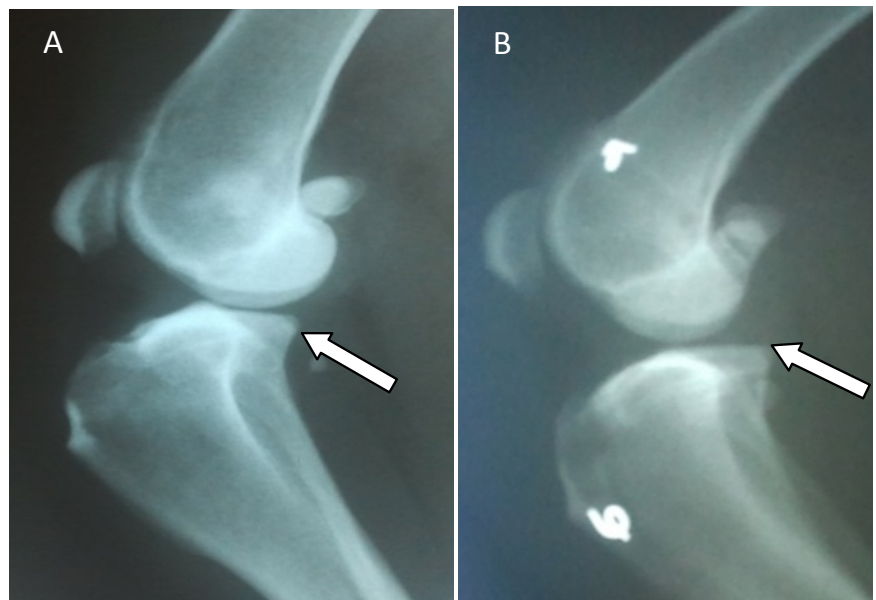

Figura 4: Imagens radiográficas mediolateral da articulação do joelho de cão com ruptura do ligamento cruzado cranial. A- observe o deslocamento cranial do platô tibial em relação ao fêmur (seta). B- Observe e presença das cavilhas de aço inoxidável na face medial da articulação do joelho e o retorno do platô tibial em relação ao fêmur (seta), após realização da técnica "TightRope" modificada.

Tabela 1: Grau de apoio do membro após 120 dias de pós-operatório e a forma de utilização do fio fluorcarbono, de acordo com a idade, peso e raça do animal

\begin{tabular}{|cccccc|}
\hline Animais & Idade/anos & Peso/kg & Raça & Fio & Nota \\
\hline 1 & 7 & 7 & SRD & Simples & 4 \\
\hline 2 & 5 & 32 & Pit Bull & Triplo & 4 \\
\hline 3 & 5 & 32 & Pit Bull & Triplo & 4 \\
\hline 4 & 4 & 14 & Beagle & Duplo & 4 \\
\hline 5 & 3 & 30 & Bulldogue Americano & Triplo & 4 \\
\hline 6 & 3 & 5 & Yorkshire & Simples & 4 \\
\hline
\end{tabular}

A rápida recuperação observada na maioria dos animais deste trabalho pode ser atribuída à técnica aqui empregada, pois de acordo com Cook et al. (2008) a técnica de "TightRope", por ser menos invasiva e extracapsular, promove o retorno rápido do paciente à locomoção, sendo relatada uma recuperação com deambulação quase normal em torno de 6 a 8 semanas. Outro fator que pode ter contribuído na recuperação dos pacientes foi a utilização do fio de fluorcarbono, um fluoropolímero termoplástico não reagente, que ao contrário do náilon, apresenta o mesmo índice de ruptura em ambiente seco ou úmido, elasticidade quase zero, não é poroso, mais rígido, e altamente resistente à abrasão (Sousa, 2009), conferindo boa adaptação e aplicabilidade, com ausência clínica de reação biológica dos pacientes aos implantes.

Já o retardo no apoio do membro dos dois animais com menos de 10 quilos observado neste relato, pode ser justificado pelo baixo peso destes pacientes ( 5 e $7 \mathrm{~kg}$ ), já que sabidamente animais com baixo peso corporal adaptam-se bem à condição de caminhar somente com três patas, quando impossibilitados de utilizar algum dos membros pélvicos, distribuindo grande parte da carga aos membros torácicos (Bastian., 2013). Além deste fato, ao exame clínico, a articulação demonstrou-se estável e sem sensibilidade dolorosa à manipulação, indicando estabilização adequada do joelho.

Cook et al. (2008) relataram que o uso da técnica de "TightRope" na estabilização da articulação do joelho de cães com ruptura do LCCr pode ser executada com sucesso apenas em pacientes de raças médias, grandes e gigantes, ou seja, pacientes com peso igual ou superior a $15 \mathrm{~kg}$, devido à necessidade de serem criados grandes túneis ósseos para a passagem e 
fixação dos implantes. Já a técnica aqui modificada, substituindose a fibra fiberware $\AA$, pelo fio de fluocarbono, foi efetiva na estabilização do joelho de animais com menos de 10 quilos, pois o menor diâmetro e a resistência do fio utilizado permitiram adequar os implantes ao tamanho dos pacientes.

Vale ressaltar que um dos animais, após cinco meses de pós-operatório, apresentou-se ao Hospital Veterinário - UFU com claudicação aguda do membro operado, demonstrando ao exame físico específico instabilidade articular, com teste de gaveta cranial e compressão tibial positivos, sendo então encaminhado para novo procedimento cirúrgico. Durante a nova intervenção cirúrgica constatou-se a ruptura do implante de fluorcarbono, sendo este talvez insuficientemente resistente para a utilização neste animal, um Pit bull de $30 \mathrm{~kg}$, obeso com temperamento extremamente agitado e com atrofia acentuada de toda a musculatura femorotibial, o que contribuiria para uma sobrecarga no joelho, já que segundo (Slocum e Slocum, 2006) em uma articulação estável, o movimento cranial da tíbia em relação ao fêmur é neutralizado também pela musculatura, diminuindo o estrese sobre o LCCr.

De maneira semelhante, Sakamoto et al. (2013) observaram a ruptura do fio de fluorcarbono $0,7 \mathrm{~mm}$, durante o posicionamento para realização de radiografías em cadáveres caninos

\section{Referências}

ARTHREX VET SYSTEMS. Orthopaedic Products \& Medical Education. Developed in conjunction with James L. Cook, DVM, PhD, Diplomate ACVS Director, Comparative Orthopaedic Laboratory, University of Missouri, 2008.

BASTIAN, N.C. Distribuição de força estatica em cães com membros amputados. Tese (Mestrado em Cirurgia Veterinária) Universidade Federal de Santa Maria (UFSM-RS), 2013.

CANAPP, Jr. S.O. The canine stifle. Clinical Techniques in Small Animal Practice, v. 22, n. 4, p. 195-205, 2007.

CAROBBI, B.; NESS, M.G. Preliminary study evaluating tests used to diagnose canine cranial cruciate ligament failure. Journal of Small Animal Practice, Gloucester, v. 50, p. 224-226, 2009.

COMERFORD, E.J. Current thoughts on canine cranial cruciate ligament disease. In: CONGR. INTERNAZ. MULTISALA (SCIVAC) 56., 2007, Rimini Proceeding. Societá culturale italiana veterinari per animali da compangia, 2007. p.147-148.

COMERFORD, E.J. et al. Update on the aetiopathogenesis os canine cranial cruciate ligament disease. Veterinary Comparative Orthopedics and Traumatology, Stuttgart, v. 24, n. 4, p. 91-98, 2011.

CONZEMIUS, M.G. et al. Effect of surgical technique on limb function after surgery for rupture of the cranial cruciate ligament in dogs. Journal of the American Veterinary Medical Association, Schaumburg, v. 226, n. 2, p. 232-236, 2005.

COOK, J.L. TightRope surgical technique, Columbias Morning Newspaper, 2008.

COOK, J.L.; LUTHER, J. K.; BEETEM, J.; KARNES, J; COOK, C. R. Clinical comparison os a novel extracapsular stabilization procedur and tibial plateau leveling osteotomy for treatment of cranial cruciate ligament deficiency in dogs. Veterinary Surgery, $\mathrm{n}$. 39, p. 315-323, 2010 submetidos a uma modificação da técnica de "TightRope", e concluíram que esse tipo de material não apresenta a resistência necessária, diferindo dos achados observados no presente relato, onde a maioria dos animais apresentaram recuperação clínica satisfatória, sendo a modificação da técnica efetiva na estabilização da RLCCr. Acredita-se que o uso criterioso do fio fluorcarbono $0,6 \mathrm{~mm}$, utilizado duplo, ou triplo e trançado de acordo com o peso dos animais, tenha sido fundamental na capacidade do implante em resistir a tensão criada pelas cavilhas fixas ao osso.

Diante desse achado, embora apenas seis animais tenham sido acompanhados no presente relato, encontrou-se uma taxa de complicação em torno de $16 \%$, pouco superiores às taxas de complicações encontradas por Cook et al. (2010) que relataram $12,5 \%$ para "TightRope", e $17,4 \%$ na TPLO em seu estudo prospectivo.

\section{Conclusão}

A técnica TRM apresentou-se menos onerosa, de fácil exequibilidade e com eficácia clínica satisfatória, sendo efetiva na estabilização do joelho de cães entre 5 a $32 \mathrm{~kg}$ acometidos por ruptura de ligamento cruzado cranial.

DIAS, L.G.G.G. Desenvolvimento e aplicação clínica de haste intramedular bloqueada angulada no tratamento de fraturas tibiais em cães. 2009. xvii, 103 f. il. Tese (doutorado em Cirurgia Veterinária) - Faculdade de Ciências Agrárias e Veterinárias, Universidade Estadual Paulista, Jaboticabal, 2009.

DURANA, J. N. Caracterização da clinica cirúrgica da ruptura do ligamento cruzado cranial em canídeos. Dissertação. Universidade de Lisboa, graduação em Medicina Veterinária, 2009.

FISCHER, C. et al. Effects of attachment sites and joint angle at the time of lateral suture fixation on tension in the suture for stabilization of the cranial cruciate ligament deficient stifle in dogs. Veterinary Surgery, Philadelphia, v. 39, n.10, p. 334-342, 2010.

FOSSUM, T. Diseases of the joint. Small Animal Surgery (2nd ed). St. Louis, Missouri, Elsevier Mosby, p. 1023-1157, 2002.

GRIFFON, D.J. A review of the pathogenesis of canine cranial cruciate ligament disease as a Basis for future preventive strategies. Veterinary Surgery, Philadelphia, v. 39, n. 4, p. 399409, 2010.

HAYASHI, K.; FRANK, J. D.; DUBINSKY, C.; ZIIENGLING. I.I.; MARKEL, M. D.; MANLEY, P. A. A. V.; MUIR, P. Histologic changes in ruptured canine cranial cruciate ligament. Veterinary Surgery, Philadelphia, v. 32, n. 3, p. 269-277, 2003.

HULSE, D. A. The stifle joint. In: OLMSTEAD, M.L. Small animal orthopedics. St. Louis: Mosby, 1995. p. 395-416.

HULSE, D. A, et al. Determination of isometric points for placement of a lateral suture in treatment of the cranial cruciate ligament deficient stifle. Veterinary Comparative Orthopedics and Traumatology, Stuttgart, v. 23, n. 4, p. 163-167, 2010.

JERRAM, R.M.; WALKER, A.M. Cranial cruciate ligament injury in the dog: pathophysiology, diagnosis and treatment. New Zealand Veterinary Journal, Wellington, v. 51, p. 149-158, 2003. 
KEALY, J.K.; MCALLISTER, H.; GRAHEM, J.P. Bones and joints In: Diagnostic radiology and ultrasonography of the dog and cat. 5. ed. St. Louis: Elsevier Saunders. p. 351-446, 2011.

KEMPER, B.; TRAPP, S. M.; PORTO, T. F.; BARCA, F. A. Movimento de gaveta em joelhos de cães submetidos à estabilização extracapsular após secção do ligamento cruzado cranial in vitro. Cienc. Rural, Jun 2013, v. 43, n. 6, p. 1096-1101. ISSN 0103-8478.

$\mathrm{KIM}$, S.E. et al. Tibial osteotomies for cranial cruciate ligament in dogs. Veterinary Surgery, Philadelphia, v. 37, n. 2, p.111-125, 2008.

LISKA, W.D.; DOYLE, N.D. Canine total knee replacement: surgical technique and one year outcome. Veterinary Surgery, Philadelphia, v. 38 n. 5, p. 568-582, 2009.

MOORE, K.W.; READ, R.A. Rupture of cranial cruciate ligament in dogs-Part II. Diagnosis and management. Compendium on Continuing Education for the Practicing Veterinarian, Yardley, PA, v.18, n. 4, p. 381-391, 1996.

OLIVEIRA, P.R; MAMPRIM, M.J.; RAHAL, S.C; BICUDO, A.L.C. Radiografia e ultrassonografia no diagnostico da ruptura do ligamento cruzado cranial em cães. Pesquisa Veterinária Brasileira, v. 29, n. 8, p. 661-665, 2009.

PALMER, R.H. Diagnosing cranial cruciate ligament pathology. Veterinary Medicine, Lenexa, v. 41, n. , p. 414-422, 2005.

PIERMATTEI, D.L.; FLO, G.L.; DECAMP, C.E. The stifle joint. Brinker, Piermattei, and Flo's Handbook of Small Animal Orthopedics and Fracture Repair (4.ed.). Philadelphia, PA, Saunders, p. 562-632, 2006.

ROOSTER, H.D.; BRUIN, T.D; BREE, H.V. Morphology and function of the crucie-te ligaments. In: MUIR, P. Advances in the canine cranial cruciate ligament. Ames, lowa: ACVS foundation e Wiley-Blackwell, 2010.
SAKAMOTO, B.Y.; ZANIBONI, L.; RUSSO, C. Estabilização da articulação femorotibiopatelar em cães por meio da utilização dos implantes "Tightrope". In. VI MOSTRA INTERNA DE TRABALHOS DE INICIAÇÂO CIENTIFICA, 23 Marilia, 2012. Anais Eletrônico, Marília 2012.

SCHULZ, K.; STIFLE. Cranial cruciate rupture ligament rupture. In: FOSSUM, T.W. Manual of small animal surgery. 3. ed. St. Louis: Mosby, 2007. p. 1254-1275.

SLOCUM, B. e SLOCUM, T. D. Cranial Cruciate Ligament. Slocum Enterprises, Inc. Disponível em (http://www. slocumenterprises.com/Articles/cranial_cruciate_ligament.htm). Acesso em: 2 de dezembro 2012.

SOUSA, M. E. Linhas 2009. http://www.tucunazul.com.br/ pdf/49c19063edae5.pdf Acesso em 22 de out. de 2013.

SOUZA, M. M. D.; RAHAL, S. C.; PADOVANI, C. R.; MAMPRIM, M. J.; CAVINI, J. H. Afecções ortopédicas dos membros pélvicos em cães: estudo retrospectivo. Ciência Rural, v. 41, p. 852-857, 2011.

TONKS C.A, et al. The effects of extra-articular suture tension on contact mechanics of the lateral compartment of cadaveric stifles treated with the TightRope CCL or lateral suture technique. Veterinary Surgery, Philadelphia, v. 39, n. 3, p. 399-409, 2010.

WHITEHAIR, J.; VASSEUR, P.B. Epidemiology of cranial cruciate ligament rupture in dogs. Journal of the American Veterinary Medical Association, v. 203, p.1016, 1993. 